of the surveillance process was confirmed, as well as the supervision and notification of cases. In addition, new physical elements were added for catheter insertion and care bundles.

Regardless of the circumstances, along with guaranteeing the availability of supplies and personnel, it is essential to guarantee the safe care of patients. The prevention and reduction of healthcare-associated infections is a urgently needed to avoid morbidity and mortality, longer hospital stays, and additional care costs due to the COVID-19 pandemic, which has required the national health system to provide timely and safe care for a significantly larger number of patients.

\section{Acknowledgments.}

Financial support. No financial support was provided relevant to this article.

Conflicts of interest. All authors report no conflicts of interest relevant to this article.

\section{References}

1. Patient Safety. World Health Organization website. https://www.who.int/ news-room/fact-sheets/detail/patient-safety. Published 2019. Accessed May 14,2021

2. The NHS patient safety strategy. Safer culture, safer systems, safer patients website. Nathional Health Service website. https://www.england.nhs.uk/wpcontent/uploads/2020/08/190708_Patient_Safety_Strategy_for_website_v4.pdf. Published 2019. Accessed May 14, 2021.

3. O'Grady NP, Alexander M, Burns LA, et al. Guidelines for the prevention of intravascular catheter-related infections, 2011. Centers for Disease Control and Prevention website. https://www.cdc.gov/infectioncontrol/pdf/ guidelines/bsi-guidelines-H.pdf. Published 2017. Accessed May 14, 2021.

4. Patel PK, Olmsted RN, Hung L, Popovich KJ, et al. A tiered approach for preventing central-line-associated bloodstream infection. Ann Intern Med 2019;171(7 suppl):S16-S22.

5. Patel PR, Weiner-Lastinger LM, Dudeck MA, et al. Impact of COVID-19 pandemic on central-line-associated bloodstream infections during the early months of 2020, National Healthcare Safety Network. Infect Control Hosp Epidemiol 2021. doi: 10.1017/ice.2021.108.

6. Molloy GJ, O'Boyle CA. The SHEL model: a useful tool for analyzing and teaching the contribution of human factors to medical error. Acad Med 2005;80:152-155.

\title{
Severe acute respiratory syndrome coronavirus 2 (SARS-CoV-2) infection in vaccinated and unvaccinated healthcare personnel in a Veterans' Affairs healthcare system
}

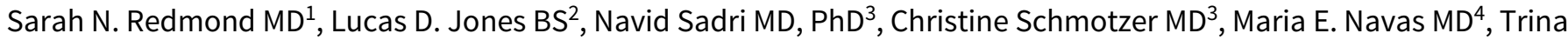 \\ F. Zabarsky $\mathrm{MSN}^{5}$, Davinder Bhullar $\mathrm{MD}^{6}$ and Curtis J. Donskey MD ${ }^{1,7}$ \\ ${ }^{1}$ Case Western Reserve University School of Medicine, Cleveland, Ohio, ${ }^{2}$ Department of Molecular Biology and Microbiology, Case Western Reserve University \\ School of Medicine, Cleveland, Ohio, ${ }^{3}$ Department of Pathology, University Hospitals Cleveland Medical Center, Cleveland, Ohio, ${ }^{4}$ Pathology and Laboratory \\ Medicine Service, Louis Stokes Cleveland Veterans' Affairs (VA) Medical Center, Cleveland, Ohio, ${ }^{5}$ Infection Control Department, Louis Stokes Cleveland VA \\ Medical Center, Cleveland, Ohio, ${ }^{6}$ Personnel Health Department, Louis Stokes Cleveland VA Medical Center, Cleveland, Ohio and ${ }^{7}$ Geriatric Research, Education, \\ and Clinical Center, Louis Stokes Cleveland VA Medical Center, Cleveland, Ohio
}

To the Editor-Coronavirus disease 2019 (COVID-19) mRNA vaccines substantially reduce but do not eliminate the risk for symptomatic and asymptomatic severe acute respiratory coronavirus virus 2 (SARS-CoV-2) infections in healthcare personnel. ${ }^{1-5}$ In a recent report, 5 (12\%) of 43 fully vaccinated personnel acquired mildly symptomatic or asymptomatic SARS-CoV-2 infection after higher-risk household exposures. ${ }^{6}$ Ongoing surveillance studies are needed to determine whethe such postvaccination "breakthrough" infections are caused by variants of concern with reduced in vitro susceptibility to neutralization by vaccine-induced antibodies. $^{7}$ Surveillance studies can also provide comparative data on COVID-19 in unvaccinated personnel.

The study protocol was approved by the Cleveland Veterans' Affairs Medical Center's institutional review board. We examined the incidence and clinical characteristics of COVID-19 in fully vaccinated versus unvaccinated personnel at the Cleveland VA

\footnotetext{
Author for correspondence: Curtis J. Donskey, E-mail: Curtis.Donskey@va.gov

Cite this article: Redmond SN, et al. (2022). Severe acute respiratory syndrome coronavirus 2 (SARS-CoV-2) infection in vaccinated and unvaccinated healthcare personnel in a Veterans' Affairs healthcare system. Infection Control \& Hospital Epidemiology, 43: 1300-1301, https://doi.org/10.1017/ice.2021.256
}

Medical Center from February 1, 2021, through May 15, 2021. Personnel were considered fully vaccinated if $>2$ weeks had passed since their second dose of the BNT162b2 vaccine. Partially vaccinated personnel with COVID-19 were excluded. Personnel health and infection control databases were reviewed to obtain information on exposure history, symptoms, and suspected transmission clusters based on contact tracing investigations. We used the Fisher exact test to compare the percentage of vaccinated versus unvaccinated employees developing COVID-19.

Nasopharyngeal respiratory samples were tested by reverse transcriptase polymerase chain reaction (RT-qPCR) for SARSCoV-2 RNA using a TaqPath COVID-19 CE-IVD RT-PCR Kit (ThermoFisher, Waltham, MA). Positive samples were further screened by multiplex qPCR for the presence of S gene L452R, E484K, N501Y mutations, S gene 69/70 deletion, and ORF1a $3675 / 3677$ deletion to identify potential variants of concern or interest. Samples with cycle threshold $<30$ were subjected to an additional multiplex RT-qPCR for a deletion in the ORF1a gene $(\text { ORF1a } \Delta 3675-3677)^{8}$ and an N501Y spike mutation. ${ }^{8,9}$ Any samples containing any of these alterations were then subjected to whole-genome sequencing for lineage identification using the 


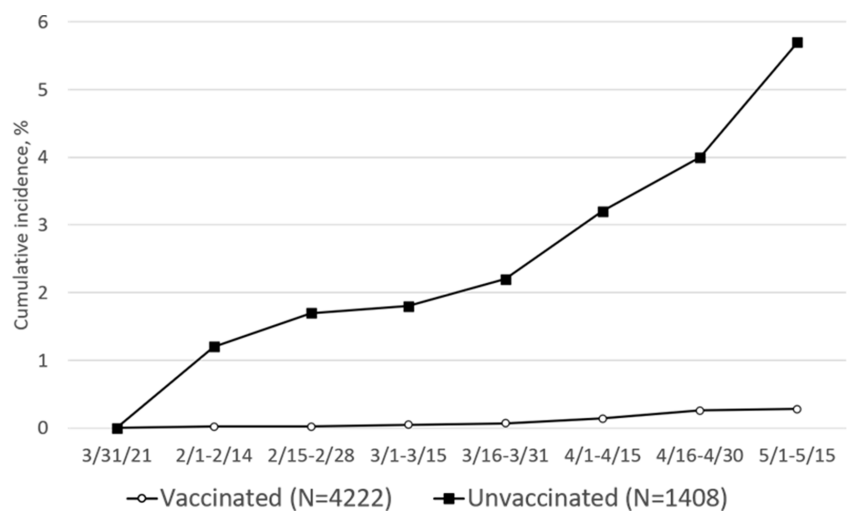

Fig. 1. Cumulative incidence of symptomatic SARS-CoV-2 infection in vaccinated versus unvaccinated healthcare personnel.

SARS-CoV-2 Research Panel and Ion Gene Studio S5 system (ThermoFisher).

Figure 1 shows the cumulative incidence of SARS-CoV-2 infections in fully vaccinated versus unvaccinated personnel. During 14 weeks of follow-up, 80 (5.7\%) of 1,408 unvaccinated employees were diagnosed with COVID-19 versus $12(0.3 \%)$ of 4,222 vaccinated employees $(P<.0001)$. Of the 12 vaccinated employees with COVID-19, 8 (67\%) reported prior higher-risk exposures, including 5 exposed to family members with SARS-CoV-2 infection, 2 exposed in the community, and 1 exposed to an unvaccinated coworker with COVID-19. All of the vaccinated employees with COVID-19 had mild symptoms and none required hospitalization. We identified 4 clusters of SARS-CoV-2 infections of 17 employees that involved suspected transmission among unvaccinated employees. No transmission clusters were linked to vaccinated personnel with COVID-19.

The mean cycle threshold values for the vaccinated employees with COVID-19 was 20 (range, 14-28). S gene dropout suggestive of the B.1.1.7 variant was detected in 7 (58.3\%) of the 12 vaccinated employees with COVID-19; the overall percentage of positive SARS-CoV-2 positive samples in the facility with $S$ gene dropout during the study period was $49 \%$ ( 107 of 217 positive tests). Whole-genome sequencing confirmed that the $S$ gene dropout specimens were lineage B.1.1.7. No other variants of concern or interest were identified.

In summary, in our facility fully vaccinated personnel were 19 times less likely than unvaccinated personnel to be diagnosed with COVID-19. The percentage of infections with the B.1.1.7 variant among vaccinated personnel was equivalent to the overall percentage of B.1.1.7 infections in our facility. No other currently classified variants of concern or interest were detected among the breakthrough infections. Most SARS-CoV-2 infections in vaccinated personnel occurred after higher-risk exposures to family members. As noted previously, Selby et $\mathrm{al}^{6}$ reported that such exposures may present a substantial risk to vaccinated individuals. Our findings provide support for recommendations that vaccinated individuals continue to take measures to avoid SARSCoV-2 exposure.

Our findings also highlight the potential for adverse consequences when personnel decline SARS-CoV-2 vaccination.
Unvaccinated personnel have a substantial ongoing risk for COVID-19. Infected personnel can transmit SARS-CoV-2 to their patients and coworkers. ${ }^{10}$ During the 14 -week study period, our healthcare system investigated 4 clusters of SARS-CoV-2 infections that involved suspected transmission by unvaccinated employees. In a long-term care facility in Kentucky, an unvaccinated employee was implicated as the source of an outbreak that resulted in infections in 20 healthcare personnel and 26 residents with 3 resident deaths. ${ }^{1}$ There are also potential adverse effects for individuals who do not develop COVID-19. In our facility, several unvaccinated individuals missed work due to quarantine after higher-risk COVID-19 exposures. Such quarantine is not required for vaccinated individuals. Facility-specific data highlighting the incidence of infections and days of work missed due to quarantine for vaccinated versus unvaccinated employees might serve as a useful reminder of the benefits of vaccination for hesitant individuals.

Acknowledgments. We thank the Personnel Health Department staff for providing data on COVID-19 infections in personnel.

Financial support. This work was supported by a Merit Review grant (no. CX001848) from the Department of Veterans' Affairs to C.J.D.

Conflicts of interest. C.J.D. has received research grants from Clorox, Pfizer, and PDI. All other authors report no conflicts of interest relevant to this article.

\section{References}

1. Cavanaugh AM, Fortier S, Lewis P, et al. COVID-19 outbreak associated with a SARS-CoV-2 R.1 lineage variant in a skilled nursing facility after vaccination program-Kentucky, March 2021. Morb Mortal Wkly Rep 2021; 70:639-643.

2. Tang L, Hijano DR, Gaur AH, et al. Asymptomatic and symptomatic SARSCoV-2 infections after BNT162b2 vaccination in a routinely screened workforce. JAMA 2021. doi: 10.1001/jama.2021.6564.

3. Keehner J, Horton LE, Pfeffer MA, et al. SARS-CoV-2 infection after vaccination in healthcare workers in California. N Engl J Med 2021;384: 1774-1775.

4. Benenson S, Oster Y, Cohen MJ, Nir-Paz R. BNT162b2 mRNA COVID-19 vaccine effectiveness among healthcare workers. N Engl J Med 2021;384: 1775-1777.

5. Angel Y, Spitzer A, Henig O, et al. Association between vaccination with BNT162b2 and incidence of symptomatic and asymptomatic SARSCoV-2 infections among healthcare workers. JAMA 2021. doi: 10.1001/ jama.2021.7152.

6. Selby L, Hewlett A, Cawcutt K, et al. Effect of SARS-CoV-2 mRNA vaccination in healthcare workers with high-risk COVID-19 exposure. Infect Control Hosp Epidemiol 2021. doi: 10.1017/ice.2021.193.

7. Hacisuleyman E, Hale C, Saito Y, et al. Vaccine breakthrough infections with SARS-CoV-2 variants. N Engl J Med 2021. doi: 10.1056/NEJMoa2105000.

8. Vogels CBF, Breban MI, Ott IM, et al. Multiplex qPCR discriminates variants of concern to enhance global surveillance of SARS-CoV-2. PLoS Biol 2021. doi: 10.1371/journal.pbio.3001236.

9. SARS-CoV-2 variant classifications and definitions. Centers for Disease Control and Prevention website. https://www.cdc.gov/coronavirus/2019ncov/cases-updates/variant-surveillance/variant-info.html. Accessed March $12,2021$.

10. Chan ER, Jones LD, Redmond SN, et al. Use of whole genome sequencing to investigate a cluster of severe acute respiratory syndrome coronavirus 2 (SARS-CoV-2) infections in emergency department personnel. Infect Control Hosp Epidemiol 2021. doi: 10.1017/ice.2021.208. 